\title{
riccafd
}

Revista Iberoamericana de Ciencias de la Actividad Fisica y el Deporte

\section{ACTIVIDAD FÍSICA Y CALIDAD DE VIDA EN LAS PERSONAS MAYORES: ESTUDIO PILOTO PQS}

\section{PHYSICAL ACTIVITY AND QUALITY OF LIFE IN ELDERLY PEOPLE: THE PQS PILOT STUDY}

Gómez-Cabello, A 1, Pardos-Mainer, E 2, González-Gálvez, N 3 y Sagarra-Romero, L 4

1 Centro Universitario de la Defensa de Zaragoza. / Grupo de investigación GENUD, Universidad de Zaragoza (España), agomez@unizar.es

2 Universidad San Jorge de Zaragoza (España), epardos@usj.es

3 Facultad del Deporte. Universidad Católica San Antonio de Murcia (España), ngonzalez@ucam.edu

4 Universidad San Jorge de Zaragoza (España), Isagarra@usj.es

Agradecimientos: El estudio "Pasos que suman: programa saludable contra el sedentarismo en las personas mayores" ha sido financiado por las ayudas a los proyectos de Buenas Prácticas de Innovación Docente-USJ (2016). Los autores también desean dar las gracias a todos los voluntarios y a los centros participantes del municipio de Villanueva de Gállego, cuya colaboración y dedicación hicieron posible este estudio.

Código UNESCO: 2312 Salud Pública

Clasificación del Consejo de Europa: 17. Otras: Actividad Física y Salud.

Correspondencia: Sagarra-Romero L. Isagarra@usj.es

DOI: http://dx.doi.org/10.24310/riccafd.2018.v7i2.5093

\section{RESUMEN}

Diferentes investigaciones muestran los efectos de la actividad física (AF) sobre la salud en los adultos mayores. Los objetivos principales de este estudio fueron: 1) evaluar la relación entre las recomendaciones de AF de la OMS y la percepción de calidad de vida en las personas mayores, y 2) valorar el efecto de un asesoramiento basado en la concienciación sobre el estilo de vida. Para ello participaron 28 adultos mayores $(70,6 \pm 6,2)$ del municipio de Villanueva de Gállego (Zaragoza), que fueron distribuidos en grupo experimental (GE) y control. Todos los sujetos completaron un cuestionario de calidad de vida (EQ-5D) y de nivel de AF (RAPA). Los sujetos que practicaban AF regular presentaron mayores niveles de percepción de calidad de vida $(p<0,05)$. La intervención basada en la concienciación podría ser una estrategia de utilidad en la mejora de la percepción de la calidad de vida de los adultos mayores.

Palabras Clave: adultos, RAPA, EQ-5D, depresión, ansiedad. 


\section{ABSTRACT}

Several investigations show the effects of physical activity (PA) on health in the older adults. The main aims of this research were: 1) to evaluate the relationship between the PA recommendations proposed by the World Health Organization and the perception of quality of life in the elderly, and 2) to determine the effect of an intervention based on lifestyle advices. For this, we selected 28 older adults (70.6 \pm 6.2$)$ from Villanueva de Gállego (Zaragoza). These participants were distributed in experimental group (EG) and control group. All subjects completed a questionnaire on quality of life (EQ-5D) and PA level (RAPA). The subjects who practiced regular PA presented higher levels of quality of life $(p<0.05)$. The intervention based on awareness could be a useful strategy in improving the perception of the quality of life of older adults.

Key Words: adults, RAPA, EA-5D, depression, anxiety.

\section{INTRODUCCIÓN}

El aumento de la esperanza de vida acaecido en las últimas décadas ha provocado un crecimiento de la población mayor en los países desarrollados. Este hecho, junto a la disminución observada en las tasas de natalidad, ha supuesto una variación de la pirámide poblacional, en la que a día de hoy se observa un incremento de la proporción de personas de edad avanzada. Datos recientes del Instituto Nacional de Estadística, en España, han mostrado que el porcentaje de población mayor de 65 años se sitúa actualmente en el 18,7\%, y además se prevé que alcance el $25,6 \%$ en 2.031 y el $34,6 \%$ en 2.066 , si se mantiene la tendencia actual ${ }^{1}$.

El envejecimiento, desde un punto de vista biológico, es la consecuencia de la acumulación de una gran variedad de daños celulares y moleculares a lo largo del tiempo, que producen un paulatino descenso de las capacidades mentales y físicas, así como un incremento del riesgo de enfermedad y discapacidad, y en última instancia, la muerte ${ }^{2}$. Esto se ve acompañado de un aumento del número de patologías en las personas mayores, entre las que se encuentran las enfermedades de tipo cardiovascular, diabetes, obesidad, osteoporosis o problemas de tipo mental como la demencia o el Alzheimer, entre otros ${ }^{3}$. Como consecuencia de estos cambios y del desarrollo de las enfermedades asociadas a estos, las personas mayores a menudo experimentan un descenso de su independencia, bienestar y por tanto, de su calidad de vida ${ }^{4-6}$. Por este motivo, y teniendo en cuenta el envejecimiento poblacional mencionado anteriormente, tratar de identificar aquellos factores modificables que se relacionan con una mejora de la calidad de vida se presenta como una necesidad de la sociedad.

En la actualidad, los estilos de vida, entre los que se encuentra la práctica de actividad física (AF), se han posicionado como importantes factores relacionados con aspectos de salud. De hecho, las evidencias científicas demuestran que 
la práctica de AF presenta importantes beneficios, previniendo y disminuyendo el riesgo de padecer diferentes enfermedades, como la obesidad, el cáncer, la hipertensión, la diabetes tipo II, la embolia, la osteoporosis o las cardiopatías isquémicas, entre otras ${ }^{7}$.

Sin embargo, y a pesar de todos los estudios científicos que corroboran los beneficios del ejercicio en este grupo de población, la práctica de AF en adultos mayores todavía es escasa. En España, según datos del Ministerio de Sanidad, Servicios Sociales e Igualdad publicados en la Encuesta Nacional de Salud "España 2011/12. Actividad física, descanso y ocio"8, las tasas de inactividad física ascienden hasta un $56,2 \%$ entre las personas de 75 a 84 años y hasta más del $76,9 \%$ entre los mayores de 85 .

Además de la inactividad física, las conductas sedentarias (actividades realizadas principalmente sentado o tumbado con un gasto energético por debajo de 1,5 METs), parecen ser por sí mismas un factor de riesgo para la salud de las personas mayores ${ }^{9}$.

Los objetivos de este estudio fueron: a) evaluar la relación entre las recomendaciones de AF establecidas por la Organización Mundial de la Salud (OMS), el tiempo dedicado a caminar y el tiempo de sedestación con la percepción de la calidad de vida en el adulto mayor; y b) valorar el efecto de un asesoramiento basado en la concienciación sobre el estilo de vida en la calidad de vida de las personas mayores.

\section{MATERIAL Y MÉTODOS}

\section{Participantes}

En este estudio piloto participaron de manera voluntaria un total de 28 personas mayores de 60 años del municipio de Villanueva de Gállego, Zaragoza (6 hombres y 22 mujeres; edad media 70,6 \pm 6,2 años), pertenecientes al proyecto titulado «Pasos que suman (PQS): programa saludable contra el sedentarismo en las personas mayores». En él se incluyó una muestra de personas mayores de 60 años no institucionalizadas residentes en un entorno rural y reclutadas a través del centro cívico, centro de salud y patronato de deportes, todos ellos pertenecientes a la localidad de Villanueva de Gállego (Zaragoza, España).

Los criterios de exclusión establecidos en este estudio fueron los siguientes: edad menor de 60 años, tener demencia o alzheimer, diagnóstico de cardiopatía severa, tratamiento en activo de quimioterapia y/o radioterapia, ser dependiente, o estar institucionalizado. El trabajo de campo del proyecto se realizó entre enero y junio de 2017. De manera previa a la participación, todos los sujetos fueron informados de los objetivos del estudio, así como de sus posibles riesgos y beneficios. Además, antes de comenzar con las pruebas, todos los participantes firmaron un consentimiento informado. 
El estudio fue aprobado por el Comité Científico de la Universidad San Jorge ( $n^{\circ}$ referencia: 006-16/17) y se realizó conforme a las directrices éticas para estudios de investigación recogidas en la declaración de Helsinki.

\section{Procedimiento}

Los participantes del estudio, una vez realizadas las pruebas que se detallarán posteriormente, fueron asignados de manera aleatoria a uno de los siguientes grupos: experimental o control. El grupo experimental $(n=18)$ recibió una charla divulgativa para fomentar un estilo de vida activo y el grupo control $(n=10)$ no recibió ninguna información.

La charla consistió en la descripción del proceso de envejecimiento y el problema del sedentarismo en este grupo de población. Además, incluyó información sobre hábitos saludables, problemas de salud derivados del sedentarismo, recomendaciones de AF establecidas por la OMS para este grupo de población, consejos sobre la práctica segura de AF y forma de cuantificar los niveles de esta. La charla, que se desarrolló en una única sesión, tuvo una duración aproximada de 40 minutos y fue impartida por los alumnos de la asignatura Actividad Física para Poblaciones con Necesidades Especiales del grado de Ciencias de la Actividad Física y el Deporte (CCAFD) (Universidad San Jorge).

Transcurridos 4 meses desde la primera evaluación, los participantes fueron citados de nuevo para realizar las pruebas y poder estudiar si se había producido algún cambio.

\section{Cuestionarios}

Todos los participantes cumplimentaron dos cuestionarios antes y después de la charla divulgativa, con el objetivo de obtener información sobre aspectos sociodemográficos, el estilo de vida y la calidad de vida autorreferida, entre otros. Los cuestionarios utilizados fueron los siguientes:

- Cuestionario EQ-5D' ${ }^{10}$ : es un instrumento que mide la calidad de vida relacionada con la salud y que puede utilizarse de manera sencilla tanto en personas sanas como en grupos con patologías. El cuestionario se divide en 5 preguntas, correspondientes a las siguientes dimensiones: movilidad, cuidado personal, actividades cotidianas, dolor/malestar y ansiedad/depresión. En cada una de ellas hay tres niveles de gravedad y el individuo debe marcar el nivel correspondiente a su estado de salud actual. Posteriormente, se pueden combinar los resultados de las 5 dimensiones para obtener un índice de calidad de vida que oscila entre 0 (la muerte) y 1 (el mejor estado de salud posible).

- Cuestionario RAPA (Rapid Assessment of Physical Activity) ${ }^{11}$. Es una herramienta validada y sencilla de administrar que permite conocer el nivel de AF de personas con una edad igual o superior a 50 años. Consta de 9 preguntas en las que la persona tiene que responder si realiza o no una 
serie de actividades. Con los resultados obtenidos de este cuestionario se puede conocer el cumplimiento o no cumplimiento de las recomendaciones semanales de AF (pregunta 6: "hago 30 minutos o más de actividades físicas moderadas por día, 5 o más días por semana".

- Cuestionario general PQS diseñado específicamente para este estudio, que incluía la siguiente información: edad, sexo, nivel educativo, estado civil, número de horas de caminar y número de horas de estar sentado. Estas dos últimas preguntas se utilizaron para definir los comportamientos activos 0 sedentarios de la siguiente manera: caminar < de 1 hora diaria (inactivo) vs. caminar 1 o más horas diarias (activo); sentado menos de 4 horas al día (no sedentario) vs. sentado 4 o más horas al día (sedentario). La definición de un comportamiento activo y sedentario se basa en análisis anteriores realizados en el proyecto multicéntrico EXERNET (estudio para la evaluación de la condición física en personas mayores, realizado en una muestra representativa de la población mayor española), en el que a través de curvas ROC se identificaron los puntos de corte a partir de los cuales se incrementaba el riesgo de padecer enfermedades relacionadas con la composición corporal, así como su relación con los niveles de condición física ${ }^{12,13}$.

\section{Mediciones antropométricas}

La talla se midió mediante el uso de un estadiómetro portátil (Seca 206, Hamburgo, Alemania). El peso corporal se midió con una báscula portátil con una capacidad máxima de $150 \mathrm{~kg}$ y un margen de error de $100 \mathrm{~g}$ (TANITA BC-601, Tanita Corp., Tokyo, Japón). Antes de la medición, los participantes debían quitarse la ropa pesada y los zapatos. El índice de masa corporal (IMC) se calculó dividiendo el peso corporal en kilogramos por la altura al cuadrado en metros. Siguiendo las indicaciones de la OMS, los valores por encima de $25 \mathrm{~kg} / \mathrm{m}^{2}$ fueron considerados como sobrepeso y los valores superiores a $30 \mathrm{~kg} / \mathrm{m}^{2}$ como obesidad.

El perímetro de cintura se midió a nivel del punto más estrecho entre la última costilla y la cresta ilíaca. Esta medida se utilizó para definir a los sujetos con obesidad central, determinada a partir de valores $\geq 88 \mathrm{~cm}$ en mujeres y de $\geq 102$ en hombres ${ }^{14}$. El perímetro de cadera se midió a nivel del máximo relieve de los músculos glúteos. Ambas medidas se realizaron mediante el uso de una cinta antropométrica flexible y no elástica (66fit).

\section{Análisis estadístico}

Se presentan valores de media y desviación estándar (DE) o de porcentajes como estadísticos descriptivos de las variables registradas. Para comprobar la normalidad de las variables de la población estudiada se utilizó el test de Kolmogorov-Smirnov. Previo a la realización de los análisis estadísticos, los datos descriptivos se han examinado con diagramas de caja para identificar posibles "outliers" en cada una de las pruebas realizadas, sin encontrarse ningún valor extremo. Como las variables utilizadas en este trabajo seguían una distribución nor- 
mal, se llevaron a cabo test paramétricos. Para ver las diferencias en la calidad de vida en función de la práctica de AF, grupos de caminar o grupos de estar sentado, se utilizó ANOVA de un factor. El efecto de la charla informativa sobre el estilo de vida de las personas mayores se realizó mediante un análisis ANOVA de medidas repetidas. Todos los análisis estadísticos se realizaron con el programa estadístico SPSS, versión 15.0. El nivel de significación se estableció en $p<0,05$.

\section{RESULTADOS}

\section{Descripción de la muestra de estudio}

La muestra de este estudio estuvo formada por un total de 28 personas (6 hombres y 22 mujeres), con una edad media de 70,6 \pm 6,2 años.

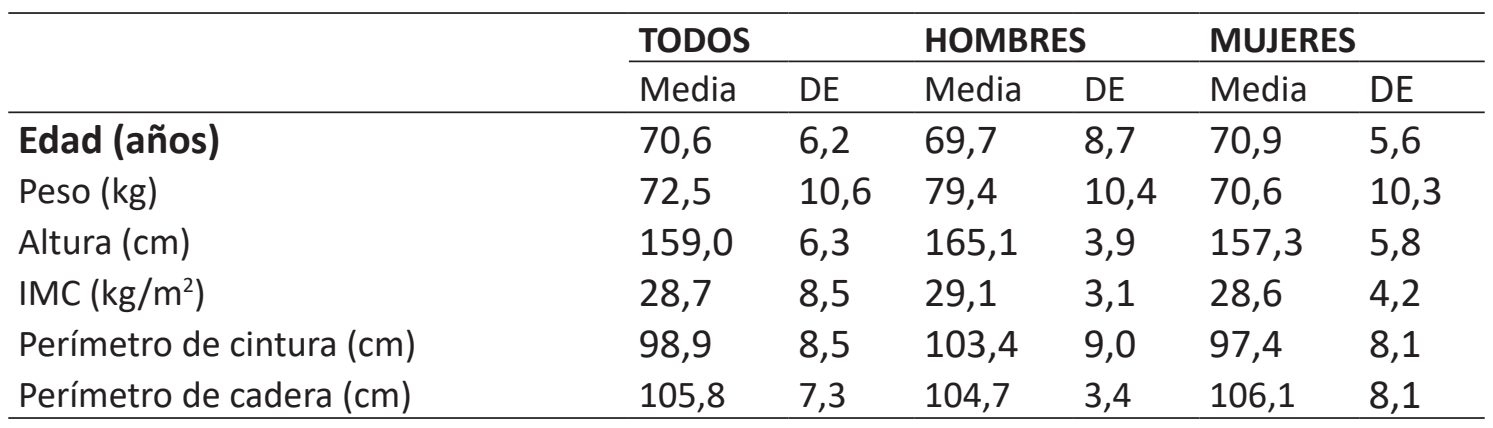

DE: Desviación estándar; IMC: Índice de masa corporal

Tabla 1. Descripción de los participantes en el proyecto Pasos Que Suman.

Atendiendo a los puntos de corte establecidos para el IMC, el 14,3\% de los mayores participantes en este estudio tenían un peso adecuado, mientras que el $42,9 \%$ tenían sobrepeso y el $42,9 \%$ obesidad.

Tomando como referencia los puntos de corte del perímetro de cintura que determinan la obesidad central ( $\geq 88$ en mujeres $y \geq 102$ en hombres), se observó que el $82,1 \%$ de la muestra de estudio tenía un exceso de grasa a nivel abdominal.

En relación con el nivel de estudios, el $60,7 \%$ de los participantes habían cursado estudios primarios, el $28,6 \%$ estudios secundarios y tan solo el $3,6 \%$ poseía un título universitario. El 7,1\% restante no poseía estudios reglados, aunque sabía leer y escribir. En cuanto al estado civil, la mayoría de los participantes estaban casados $(67,9 \%)$, mientras que un $10,7 \%$ eran solteros, un $17,9 \%$ eran viudos y un $3,6 \%$ estaban separados.

\section{Influencia de la práctica de AF sobre la calidad de vida}

Tal y como se muestra en la figura 1 , se observaron diferencias significativas en la calidad de vida entre aquellos que cumplían las recomendaciones de AF 
(al menos 30 minutos de AF moderada, 5 o más días a la semana) y los que no llegaban a los niveles mínimos de AF semanal establecidos por la OMS. Concretamente, las puntuaciones obtenidas en el índice de calidad de vida aportado por el cuestionario fueron de 0,85 $\pm 0,13$ frente al $0,71 \pm 0,21$, respectivamente; $\mathrm{p}<0,05$.

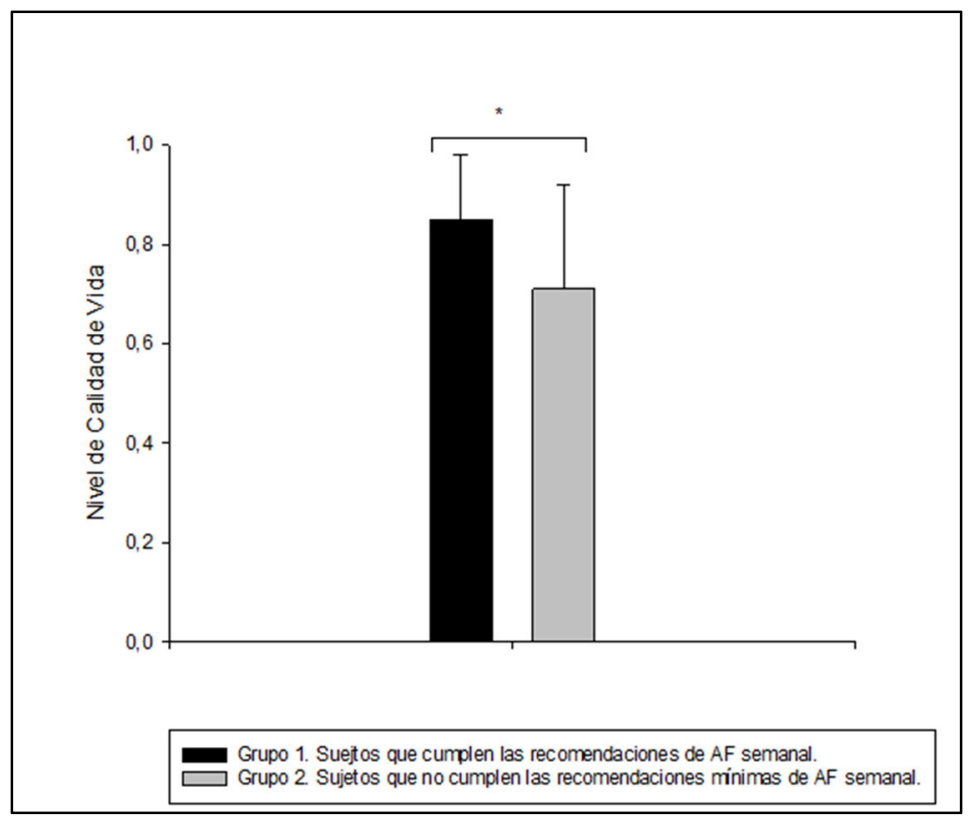

Figura 1. Calidad de vida percibida en relación con el nivel de AF semanal.

En la tabla 2 se muestran los porcentajes de respuesta en cada una de las cinco dimensiones de salud del cuestionario EQ-5D. Tal y como se observa, las mayores diferencias entre aquellos que cumplían las recomendaciones y los que no, se observaron en las dimensiones de dolor-malestar y ansiedad-depresión. Concretamente, el $71,4 \%$ de los participantes que no cumplían las recomendaciones de AF semanales tenían dolor o malestar, frente al 42,9\% de los que sí que cumplían las recomendaciones.

En relación con la ansiedad y depresión, también se observaron porcentajes más elevados entre aquellos que no cumplían las recomendaciones de AF $(42,8 \%$ vs. $28,6 \%)$.

\begin{tabular}{llll}
\hline VARIABLES & & $\begin{array}{l}\text { SI AF } \\
\text { EQ-5D }\end{array}$ & $\begin{array}{l}\text { NO AF } \\
\%\end{array}$ \\
\hline \multirow{3}{*}{ Movilidad } & No tengo problemas para caminar & 85,7 & 85,7 \\
& Tengo algunos problemas & 14,3 & 14,3 \\
& Tengo que estar en la cama & 0 & 0 \\
\cline { 2 - 4 } Cuidado personal & No tengo problemas & 100 & 92,9 \\
& Tengo algunos problemas & 0 & 7,1 \\
& Incapaz de lavarme o vestirme & 0 & 0 \\
\hline
\end{tabular}




\begin{tabular}{llll}
\cline { 2 - 4 } $\begin{array}{l}\text { Actividades } \\
\text { vida diaria }\end{array}$ & No tengo problemas & 92,9 & 92,9 \\
& Tengo algunos problemas & 7,1 & 7,1 \\
Dolor o malestar & Soy incapaz de realizarlas & 0 & 0 \\
\cline { 2 - 4 } & No tengo dolor ni malestar & 57,1 & 28,6 \\
& Tengo moderado dolor o malestar & 42,9 & 50,0 \\
Ansiedad o & Tengo mucho dolor o malestar & 0 & 21,4 \\
\cline { 2 - 4 } depresión & No estoy ansioso o deprimido & 71,4 & 57,1 \\
& Moderadamente ansioso/ deprimido & 28,6 & 35,7 \\
\hline
\end{tabular}

Tabla 2. Relación de las variables EQ-5D con las recomendaciones de AF.

\section{Influencia del tiempo de estar sentado sobre la calidad de vida}

Tal y como se aprecia en la figura 2, aquellas personas que permanecían 4 o más horas sentadas al día tenían menor calidad de vida autorreferida que aquellas que dedicaban menos de 4 horas a esta conducta sedentaria $(0,67 \pm 0,22$ vs. $0,82 \pm 0,14$, respectivamente; $p<0,05)$.

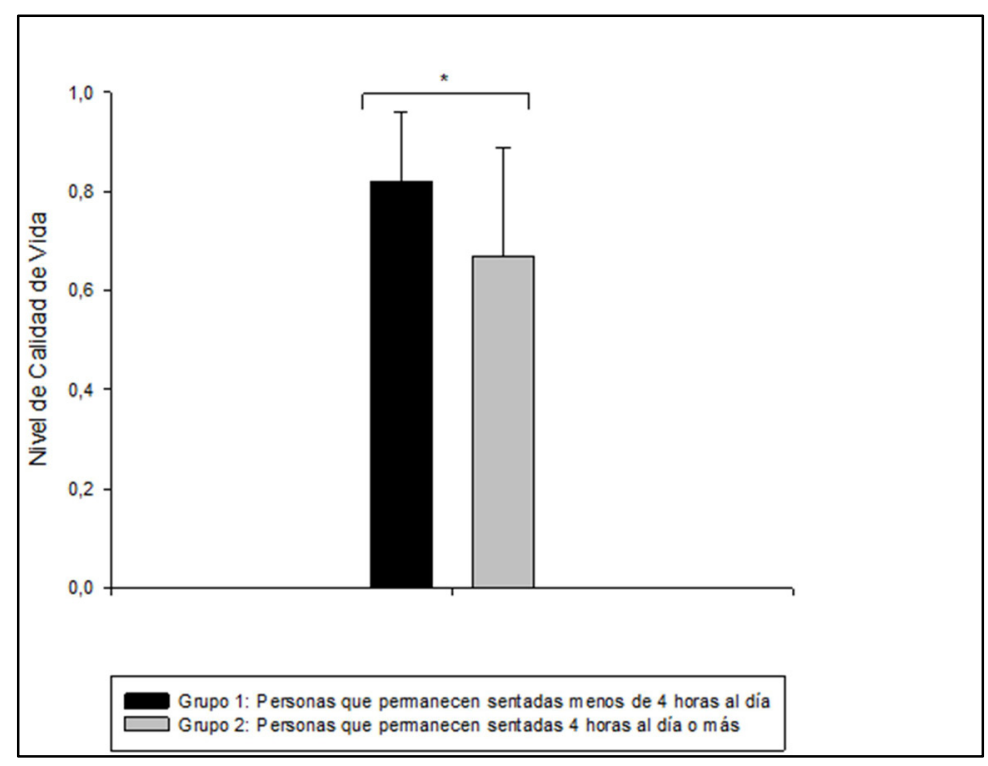

Figura 2. Calidad de vida percibida en relación con las horas diarias de estar sentado.

Los resultados de la tabla 3 muestran como el porcentaje de personas con problemas de movilidad, problemas para el desempeño de las actividades de la vida diaria, dolor o malestar y ansiedad o depresión es mayor entre aquellos que permanecen sentados 4 o más horas al día.

\begin{tabular}{llll}
\hline $\begin{array}{l}\text { VARIABLES } \\
\text { EQ-5D }\end{array}$ & & $\begin{array}{l}<4 \mathrm{~h} / \text { día } \\
\mathbf{\%}\end{array}$ & $\begin{array}{l}\mathbf{2 4} \text { h/día } \\
\mathbf{\%}\end{array}$ \\
\hline \multirow{3}{*}{ Movilidad } & No tengo problemas para caminar & 94,1 & 70,0 \\
& Tengo algunos problemas & 5,9 & 30,0 \\
& Tengo que estar en la cama & 0 & 0 \\
\hline
\end{tabular}




\begin{tabular}{llll} 
& No tengo problemas & 94,1 & 100 \\
Cuidado personal & Tengo algunos problemas & 5,9 & 0 \\
& Incapaz de lavarme o vestirme & 0 & 0 \\
\cline { 2 - 4 } Actividades & No tengo problemas & 100 & 80 \\
vida diaria & Tengo algunos problemas & 0 & 20 \\
& Soy incapaz de realizarlas & 0 & 0 \\
\cline { 2 - 4 } Dolor o malestar & No tengo dolor ni malestar & 47,1 & 30,0 \\
& Tengo moderado dolor o malestar & 47,1 & 50,0 \\
Ansiedad o & Tengo mucho dolor o malestar & 5,9 & 20,0 \\
\cline { 2 - 4 } depresión & No estoy ansioso o deprimido & 76,5 & 40,0 \\
& Moderadamente ansioso/ deprimido & 23,5 & 50,0 \\
& Estoy muy ansioso o deprimido & 0 & 10,0 \\
\hline
\end{tabular}

Tabla 3. Relación de las variables EQ-5D con los grupos de estar sentado.

\section{Influencia del tiempo dedicado a caminar sobre la calidad de vida}

En la figura 3 se muestran las puntuaciones de calidad de vida de aquellas personas que caminaban menos de 1 hora diaria y de aquellas que caminaban 1 hora o más. No se observaron diferencias significativas en este parámetro entre los dos grupos de caminar.

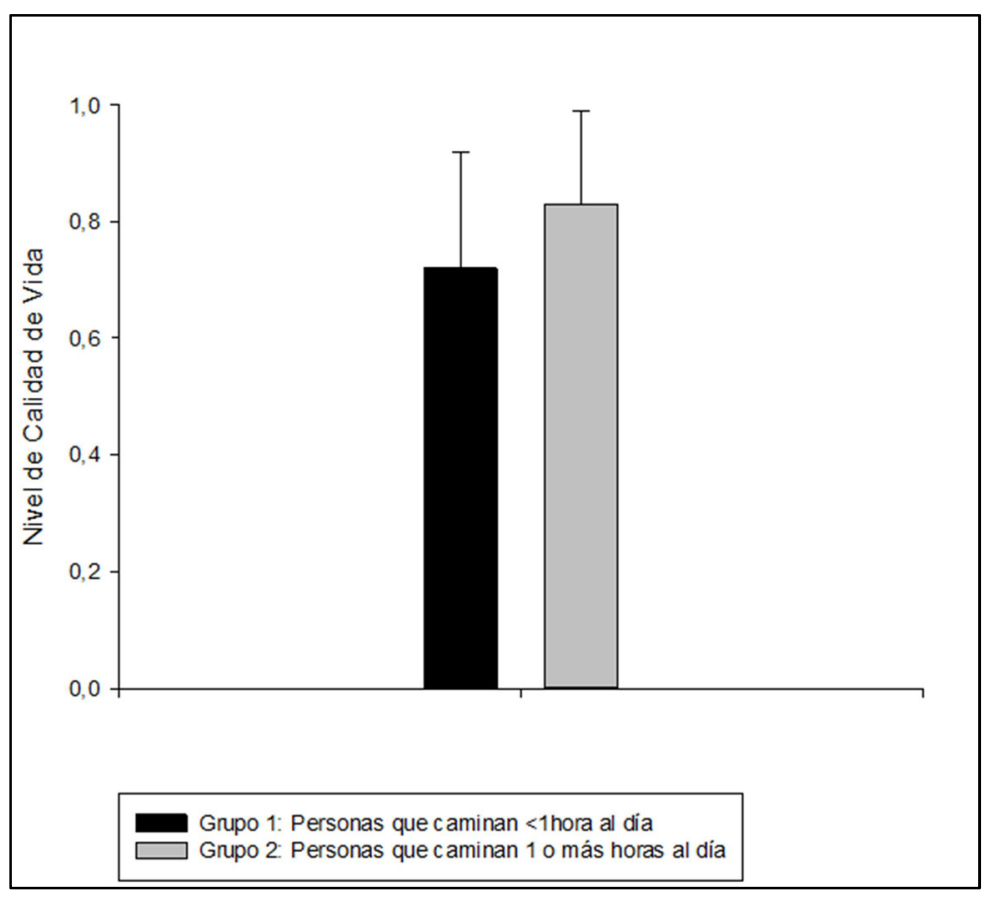

Figura 3. Calidad de vida percibida en relación con las horas diarias de caminar.

En relación a los grupos de caminar y los resultados obtenidos en el cuestionario EQ-5D, se observa que la dimensión más afectada por la inactividad es la del dolor y malestar, ya que el porcentaje de personas que reportan tener dolores y malestar es mayor entre aquellos participantes que caminan menos de 1 hora al día. 


\begin{tabular}{|c|c|c|c|}
\hline $\begin{array}{l}\text { VARIABLES } \\
\text { EQ-5D }\end{array}$ & & $\begin{array}{l}<1 \text { h/día } \\
\%\end{array}$ & $\begin{array}{l}\geq 1 \text { h/día } \\
\%\end{array}$ \\
\hline \multirow{3}{*}{ Movilidad } & No tengo problemas para caminar & 85,7 & 85,7 \\
\hline & Tengo algunos problemas & 14,3 & 14,3 \\
\hline & Tengo que estar en la cama & 0 & 0 \\
\hline \multirow{3}{*}{ Cuidado personal } & No tengo problemas & 92,9 & 100 \\
\hline & Tengo algunos problemas & 7,1 & 0 \\
\hline & Incapaz de lavarme o vestirme & 0 & 0 \\
\hline \multirow{3}{*}{$\begin{array}{l}\text { Actividades } \\
\text { vida diaria }\end{array}$} & No tengo problemas & 92,9 & 92,9 \\
\hline & Tengo algunos problemas & 7,1 & 7,1 \\
\hline & Soy incapaz de realizarlas & 0 & 0 \\
\hline \multirow{3}{*}{ Dolor o malestar } & No tengo dolor ni malestar & 21,4 & 64,3 \\
\hline & Tengo moderado dolor o malestar & 64,3 & 28,6 \\
\hline & Tengo mucho dolor o malestar & 14,3 & 7,1 \\
\hline \multirow{3}{*}{$\begin{array}{l}\text { Ansiedad o } \\
\text { depresión }\end{array}$} & No estoy ansioso o deprimido & 64,3 & 64,3 \\
\hline & Moderadamente ansioso/ deprimido & 28,6 & 35,7 \\
\hline & Estoy muy ansioso o deprimido & 7,1 & 0 \\
\hline
\end{tabular}

Tabla 4. Relación de las variables EQ-5D con los grupos de caminar.

\section{Efecto de la charla informativa sobre la calidad de vida}

En la tabla 5 se muestran los valores obtenidos en el índice de calidad de vida del cuestionario EQ-5D antes y después de la charla informativa impartida por los alumnos de la asignatura Actividad Física para Poblaciones con Necesidades Especiales de CCAFD. Tal y como se observa, en ninguno de los dos grupos se observaron diferencias significativas entre las evaluaciones "antes" y "después" (ambos $p>0,05$ ); sin embargo, aunque no tuviera significación estadística, el grupo que recibió la charla mejoró su puntuación en el índice de calidad de vida en un $9,1 \%$, mientras que el grupo que no la recibió disminuyó esta puntuación en un $22,8 \%$.

\begin{tabular}{|c|c|c|c|c|c|c|}
\hline \multirow{2}{*}{ CALIDAD DE VIDA } & \multicolumn{2}{|l|}{ ANTES } & \multicolumn{2}{|c|}{ DESPUÉS } & \multirow{2}{*}{$\begin{array}{l}\% \text { de } \\
\text { cambio }\end{array}$} & \multirow[b]{2}{*}{$p$} \\
\hline & Media & $\mathrm{DE}$ & Media & $\mathrm{DE}$ & & \\
\hline Grupo sin charla informativa & 0,73 & 0,21 & 0,55 & 0,37 & $-22,8$ & 0,250 \\
\hline Grupo con charla informativa & 0,79 & 0,17 & 0,79 & 0,28 & 9,1 & 0,947 \\
\hline
\end{tabular}

Tabla 5. Calidad de vida percibida en los participantes relacionada con la charla informativa.

\section{DISCUSIÓN}

Los principales hallazgos de este estudio piloto fueron: 1) el cumplimiento de las recomendaciones semanales de AF moderada, así como permanecer sentado menos de 4 horas al día, se asocia con una mejor calidad de vida en las personas mayores; 2) el dolor-malestar y la ansiedad-depresión son las dimen- 
siones sobre las que existe una mayor influencia de la AF y el sedentarismo en la población mayor; y 3) la implementación de una charla informativa podría ser de utilidad para lograr una mejora de la calidad de vida mediante la AF.

La calidad de vida de nuestro día a día equivale a la calidad de nuestro envejecimiento ${ }^{15}$. De acuerdo con los resultados obtenidos en este estudio piloto, se determinó que existía una relación entre la calidad de vida y la AF semanal; es decir, que aquellos que cumplían las recomendaciones mínimas de AF presentaban mejores niveles de calidad de vida. Estos resultados están en consonancia con otras investigaciones en las que se ha mostrado una mayor calidad de vida, salud percibida, bienestar psicológico, estado de ánimo y una menor depresión en los sujetos que participan en $\mathrm{AF}^{16-18}$.

En relación a este hecho, es conocido que la práctica regular de AF repercute positivamente sobre la salud de las personas mayores ${ }^{19}$, disminuyendo el riesgo cardiovascular ${ }^{20}$, mejorando el control de patologías crónicas (diabetes, hipertensión arterial, etc. $)^{21,22}$, reduciendo el riesgo de mortalidad y obesidad ${ }^{23}$, ${ }^{24}$, mejorando las funciones cognitivas ${ }^{25}$ y disminuyendo el número de caídas y fracturas óseas ${ }^{26}$. Por ello, las estrategias encaminadas a aumentar la práctica de AF de las personas mayores, como la charla informativa realizada en el marco de este proyecto, pueden tener repercusiones positivas en la concienciación sobre los efectos beneficiosos que logra la AF y en última instancia, aumentar la adherencia al ejercicio durante el envejecimiento.

De hecho, el grupo que fue asesorado mediante una charla informativa sobre hábitos saludables y fomento de estilo de vida activo, mostró un ligero cambio positivo sobre su calidad de vida; sin embargo, el grupo que no recibió este "input" mostró un empeoramiento de esta variable. Así mismo, se mostraron valores más bajos en el grupo que no recibió la charla informativa respecto al que la recibió $(0,55$ vs 0,79$)$, a pesar de que estos no fueran significativos. Teniendo en cuenta el descenso de la calidad de vida en el grupo que no recibió la charla, el ligero aumento de la calidad de vida observado en el grupo que la recibió se considera como un resultado positivo de la intervención de este estudio.

En la actualidad se conoce que además del nivel de AF, el sedentarismo también tiene un papel fundamental sobre la salud de las personas, incluidas las de avanzada edad $^{9}$, por lo que los resultados de nuestro estudio van en línea con las investigaciones publicadas hasta la fecha. A este respecto, los datos obtenidos en este proyecto mostraron que aquellas personas que permanecían sentadas 4 o más horas al día tenían una calidad de vida peor que las personas que permanecían sentadas menos tiempo. A pesar de estos resultados, a día de hoy se conoce que alrededor del $60 \%$ de los adultos pasan sentados más de 4 horas al día ${ }^{27}$. Por este motivo, parece necesario tratar de reducir las conductas sedentarias de la población, con el objetivo de mejorar su estado de salud y calidad de vida.

La principal limitación de este estudio piloto es el tamaño de la muestra, así como el género, ya que la mayoría de los participantes eran mujeres. Sin em- 
bargo, una vez comprobada la viabilidad del proyecto, así como la implicación de los alumnos de la asignatura Actividad Física para Poblaciones con Necesidades Especiales del grado de CCAFD, se pretende seguir con el reclutamiento de participantes para aumentar de manera significativa el tamaño de la misma.

\section{CONCLUSIONES}

Las personas mayores que cumplen las recomendaciones de AF establecidas por la OMS, así como las que permanecen sentadas menos de 4 horas al día, refieren una mayor calidad de vida. El dolor, el malestar, la ansiedad y la depresión son las dimensiones relacionadas con la calidad de vida que parecen tener un mayor beneficio a través de la práctica de AF regular y la reducción de las conductas sedentarias. La implementación de charlas informativas podría ser una estrategia de utilidad en la mejora de la calidad de vida de las personas mayores, probablemente debido al cambio en los estilos de vida que se presupone que ocurre con este tipo de actividad.

\section{REFERENCIAS}

1. Instituto Nacional de Estadística (INE). Proyección de Población 2016-2066. Madrid: INE; 2017. Disponible en: http://www.ine.es/prensa/np994.pdf.

2. Organización Mundial de la Salud (OMS). Envejecimiento y salud. 2015. Disponible en: http://www.who.int/mediacentre/factsheets/fs404/es/.

3. Noriega Borge MJ, García Hernández M, M.P. TE. Proceso de envejecer: cambios físicos, cambios psíquicos, cambios sociales. Comunidad Autónoma de Cantabria: Universidad de Cantabria; 2005.

4. Reyes I, Castillo CJA. El envejecimiento humano activo y saludable, un reto para el anciano, la familia, la sociedad. Rev Cubana Invest Biomed. 2011;30(3):354-9.

5. Dorantes-Mendoza G, Ávila-Funes JA, Mejía-Arango S, Gutiérrez-Robledo LM. Factores asociados con la dependencia funcional en los adultos mayores: un análisis secundario del Estudio Nacional sobre Salud y Envejecimiento en México. Rev Panam Salud Pública. 2007;22(1):1-11.

6. Aguilar JM, Álvarez J, Lorenzo JJ. Factores que determinan la calidad de vida de las personas mayores. INFAD. 2011;1(4):161-8.

7. Vogel T, Brechat PH, Lepretre PM, Kaltenbach G, Berthel M, Lonsdorfer J. Health benefits of physical activity in older patients: a review. Int J Clin Pract. 2009;63(2):30320.

8. Ministerio de Sanidad, Servicios Sociales e Igualdad. Encuesta Nacional de Salud España 2011/12. Actividad física, descanso y ocio. Serie Informes monográficos $n^{\circ}$ 4. Madrid: Ministerio de Sanidad, Servicios Sociales e Igualdad; 2014.

9. de Rezende LF, Rodrigues Lopes M, Rey-Lopez JP, Matsudo VK, Luiz Odo C. Sedentary behavior and health outcomes: an overview of systematic reviews. PLoS One. 2014;9(8):e105620. 
10. Herdman M, Badia X, Berra S. [EuroQol-5D: a simple alternative for measuring health-related quality of life in primary care]. Aten Primaria. 2001;28(6):425-30.

11. Topolski TD, LoGerfo J, Patrick DL, Williams B, Walwick J, Patrick MB. The Rapid Assessment of Physical Activity (RAPA) among older adults. Prev Chronic Dis. 2006;3(4):A118.

12. Gomez-Cabello A, Pedrero-Chamizo R, Olivares PR, Hernandez-Perera R, RodriguezMarroyo JA, Mata E, et al. Sitting time increases the overweight and obesity risk independently of walking time in elderly people from Spain. Maturitas. 2012;73(4):337-43.

13. Morales S, Gomez-Cabello A, Gonzalez-Aguero A, Casajus JA, Ara I, Vicente-Rodriguez G. [Sedentarism and physical fitness in postmenopausal women]. Nutr Hosp. 2013;28(4):1053-9.

14. Lean ME, Han TS, Morrison CE. Waist circumference as a measure for indicating need for weight management. BMJ. 1995;311(6998):158-61.

15. González U, Grau J, Amarillo M. La calidad de vida como problema de la bioética. Sus particularidades en la salud humana. JR A, editor. Ciudad de la Habana1997. 279-85 p.

16. Serrano-Sanchez JA, Lera-Navarro A, Espino-Torón L. Actividad física y diferencias de fitness funcional y calidad de vida en hombres mayores / Physical activity and differences of functional fitness and quality of life in older males. RIMCAFD. 2013;13(49):87-105.

17. De Juanas, Á. Limón MR, Navarro E. Análisis del bienestar psicológico, estado de salud percibido y calidad de vida en personas adultas mayores. Pedagogía Social. Revista Interuniversitaria. 2013;22:153-68.

18. Medina-Porqueres, I. Romero-Galisteo, RP. Gálvez-Ruiz, P. Moreno-Morales, N. Sánchez-Guerrero, E. Cuesta-Vargas, JS. et al. Alexitimia y depresión en mayores que practican actividad física dirigida. Revista Iberoamericana de Ciencias de la Actividad Física y el Deporte. 2016;5(3):36-48.

19. Taylor D. Physical activity is medicine for older adults. Postgrad Med J. 2014;90(1059):26-32.

20. Eijsvogels TM, Molossi S, Lee DC, Emery MS, Thompson PD. Exercise at the Extremes: The Amount of Exercise to Reduce Cardiovascular Events. J Am Coll Cardiol. 2016;67(3):316-29.

21. Warburton DE, Katzmarzyk PT, Rhodes RE, Shephard RJ. Evidence-informed physical activity guidelines for Canadian adults. Can J Public Health. 2007;98 Suppl 2:S1668.

22. Hu G, Qiao Q, Silventoinen K, Eriksson JG, Jousilahti P, Lindstrom J, et al. Occupational, commuting, and leisure-time physical activity in relation to risk for Type 2 diabetes in middle-aged Finnish men and women. Diabetologia. 2003;46(3):322-9.

23. Vincent HK, Raiser SN, Vincent KR. The aging musculoskeletal system and obesityrelated considerations with exercise. Ageing Res Rev. 2012;11(3):361-73.

24. Gregg EW, Cauley JA, Stone K, Thompson TJ, Bauer DC, Cummings SR, et al. Relationship of changes in physical activity and mortality among older women. JAMA. 2003;289(18):2379-86.

25. Barnes JN. Exercise, cognitive function, and aging. Adv Physiol Educ. 2015;39(2):5562. 
26. Sherrington C, Whitney JC, Lord SR, Herbert RD, Cumming RG, Close JC. Effective exercise for the prevention of falls: a systematic review and meta-analysis. J Am Geriatr Soc. 2008;56(12):2234-43.

27. Harvey JA, Chastin SF, Skelton DA. Prevalence of sedentary behavior in older adults: a systematic review. Int J Environ Res Public Health. 2013;10(12):6645-61.

Referencias totales citadas: 27

Referencias citadas correspondientes a la Rev Ib CC Act Fis Dep: 1 